\title{
We need clear health messages about exercise
}

We regret that during the processing for publication of this letter by Hupin and colleagues (BMJ 2016;355:6252, doi: http://dx. doi.org/10.1136/bmj.i6252) the name of the third author was misspelled online and in print.

The third author is Professor Vincent Gremeaux [not Gremaux, as published].
In addition, Professor Gremeaux's affiliation should have read "Rehabilitation Department, CIC INSERM 1432, Technological Platform and UMR INSERM 1093, CAPS (Cognition, Action, et Plasticité Sensorimotrice), University Hospital of Dijon, Dijon, France" [not "Rehabilitation Department, CIC INSERM 1432, Technological Platform, University Hospital of Dijon, Dijon, France," as published]. 